\section{SINGLE-CELL RNA-SEQ REVEALS THE CRITICAL ROLES OF THE STING- AND MDA5-MEDIATED CYTOSOLIC NUCLEIC ACID-SENSING PATHWAYS AS WELL AS IFNAR/STAT2 SIGNALING IN RECOMBINANT MVA- INDUCED ANTITUMOR IMMUNITY}

${ }^{1}$ Shuaitong Liu*, ${ }^{1}$ Gregory Mazo, ${ }^{2}$ Tuo Zhang, ${ }^{1}$ Ning Yang, ${ }^{1} Y i$ Wang, ${ }^{2}$ Adrian Tan, ${ }^{3}$ Jiahu Wang, ${ }^{4} J o h n$ Choi, ${ }^{2} J e n n y$ Zhaoying Xiang, ${ }^{1}$ Taha Merghoub, ${ }^{1}$ Jedd Wolchok, ${ }^{1}$ Liang Deng. 'Memorial Sloan Kettering Cancer Center, New York, NY, United States; ${ }^{2}$ Weill Cornell Medical College, New York, NY, United States; ${ }^{3}$ Genvira Biosciences, Ottawa, Canada; ${ }^{4}$ IMVAQ Therapeutics, New York, NY, United States

Background Oncolytic viruses are promising therapeutic agents for advanced cancers because of their ability to induce antitumor innate and adaptive immunity. Modified vaccinia virus Ankara (MVA) is an attenuated, replication-deficient poxvirus safe for human use, making it a favorable platform for cancer immunotherapy. Previously we discovered the E5R gene in MVA encodes an inhibitor of the cGAS/STING-mediated cytosolic DNA-sensing pathway. The engineered MVA deleting the E5R gene and expressing a dendritic cell (DC) growth factor Flt3L together with a $\mathrm{T}$ cell co-stimulator OX40L generates strong antitumor immunity when delivered intratumorally. We also demonstrated that intratumoral (IT) injection of 1st generation rMVA promotes CD8 + activation in a cGAS/STINGand STAT1/STAT2-dependent manner and depletes the OX40hi regulatory $\mathrm{T}$ cells (Tregs) via OX40L/OX40 interaction and IFNAR signaling. The E3L gene from MVA encodes an inhibitor of the cytosolic dsRNA-sensing pathways. The purpose of this study is to investigate whether rMVA $\triangle \mathrm{E} 3 \mathrm{~L}$, with the deletion of E3L from rMVA (MVA $\triangle E 5 \mathrm{R}-\mathrm{hFlt3L}$ mOX40L), further improves its antitumor effects. We also focused on utilizing single-cell RNA-seq (scRNA-seq) to investigate the alteration of the immunosuppressive tumor microenvironment by IT delivery of rMVA $\triangle \mathrm{E} 3 \mathrm{~L}$.

Methods We engineered rMVA $\triangle \mathrm{E} 3 \mathrm{~L}$ by deleting the E3L gene from rMVA. We investigated the innate immune responses of bone marrow-derived DCs (BMDCs) and tumor cell lines to rMVA $\triangle E 3 \mathrm{~L}$ and rMVA. We also compared the antitumor efficacy of the two viruses in murine B16-F10 melanoma and MC38 colon cancer models. In addition, we performed scRNA-seq of sorted tumor-infiltrating CD45+ immune cells from tumors harvested two days after IT injection with rMVA $\triangle \mathrm{E} 3 \mathrm{~L}$ or PBS in wild-type, MDA5/STING-, STAT2-, or IFNAR1-deficient mice.

Results Compared with rMVA, rMVA $\triangle \mathrm{E} 3 \mathrm{~L}$ more potently activates both the cGAS/STING- and MDA5/MAVS-mediated nucleic acid-sensing pathways in DCs and tumor cell lines. IT rMVA $\triangle E 3 L$ generates stronger antitumor effects than rMVA. Our results showed that after rMVA $\triangle \mathrm{E} 3 \mathrm{~L}$ treatment, there was an influx of inflammatory monocytes into the tumors in WT mice, absent in STAT2 or IFNAR knockout mice. Evaluation of viral transcriptomes at a single-cell level revealed that macrophages and monocytes were more susceptible to rMVA$\triangle \mathrm{E} 3 \mathrm{~L}$ than other cell types. The transcriptomic changes on DC subsets are largely dependent on IFNAR/STAT2 signaling. By contrast, MDA5/STING-mediated pathway determines the transcriptome profile of inflammatory monocytes in response to $\mathrm{rMVA} \Delta \mathrm{E} 3 \mathrm{~L}$.

Conclusions Taken together, our results demonstrate that scRNA-seq is a powerful approach to interrogate the host immune responses to immunogenic viruses, which would guide future designs of more effective viral-based cancer immunotherapy.
Acknowledgements This work was supported that the Society of Memorial Sloan Kettering (MSK) research grant (L.D.), MSK Technology Development Fund (L.D.), Parker Institute for Cancer Immunotherapy Career Development Award (L.D.), sponsored research award from IMVAQ Therapeutics. This work was supported in part by the Swim across America (J.D. W., T.M.), Ludwig Institute for Cancer Research (J.D.W., T. M.), National Cancer Institute grants R01 CA56821 (J.D.W). This research was also funded in part through the NIH/NCI Cancer Center Support Grant P30 CA008748.

http://dx.doi.org/10.1136/jitc-2021-SITC2021.696 